
\title{
$\angle S$ Research Square \\ The therapeutic potential and deleterious effect of glucocorticoids on AOM/DSS-induced colorectal cancer in mice
}

Jun Pu

Xi'an Jiaotong University Medical College First Affiliated Hospital

Xinrui Zhou

Xi'an Jiaotong University Medical College First Affiliated Hospital

Jiaxin Liu

Xi'an Jiaotong University Medical College First Affiliated Hospital

Peng Hou

Xi'an Jiaotong University Medical College First Affiliated Hospital

Meiju Ji ( $\triangle$ mjji0409@163.com )

Xi'an Jiaotong University Medical College First Affiliated Hospital

\section{Research}

Keywords: Dexamethasone, Colitis-associated colorectal cancer, Inflammatory bowel disease, Colitis, TNFa

Posted Date: March 22nd, 2021

DOl: https://doi.org/10.21203/rs.3.rs-323797/v1

License: (c) (1) This work is licensed under a Creative Commons Attribution 4.0 International License. Read Full License 


\section{Abstract \\ Background}

Glucocorticoids (GCs) are widely used in the treatment of various autoimmune and inflammatory diseases, including inflammatory bowel disease (IBD), but the effect of GCs on the development of colitisassociated colorectal cancer (CAC) is largely undefined.

\section{Methods}

We first established azoxymethane and dextran sulfate sodium (AOM/DSS)-induced colorectal cancer and DSS-induced colitis in mice. Dexamethasone (DEX) was then administered at different periods of time to determine its effect on tumorigenesis and tumor progression. Moreover, body weight, stool property and fecal blood of mice were recorded. At the end of the study, the number and load of tumors were evaluated, and the expression of proteins associated with cell proliferation were measured. To evaluate the inflammation in colon, we detected the level of pro-inflammatory cytokine TNFa, and mucosal infiltration of inflammatory cells. Meanwhile, we also assessed the activity of MAPK/JNK pathway.

\section{Results}

AOM injection followed by three cycles of drinking water containing 1.5\% DSS successfully induced multiple tumor formation in mouse colon and rectum. Both early and late DEX intervention suppressed tumor growth in mouse colorectum and significantly downregulated the expression of PCNA and cyclin D1. Meanwhile, DEX treatment significantly inhibited the TNFa production, mucosal infiltration of inflammatory cells and the activity of JNK pathway, which was more prominent in mice with early DEX intervention. However, DEX treatment deteriorated the general state of mouse manifested by greater loss of body weight and rectal bleeding.

\section{Conclusions}

Our data conclude that both early and late DEX intervention significantly ameliorate colonic inflammation and inhibit the development of AOM/DSS-induced colorectal cancer, at least partly due to the inhibition of MAPK/JNK pathway. However, the deleterious effect on the general condition of mouse may limit the duration of GCs treatment.

\section{Background}

Colorectal cancer (CRC) is one of the most common malignancies in the world with the third incidence and the second mortality rate [1]. Various factors contribute to the initiation and progression of CRC. 
Chronic inflammation is one of the most important factors, which has been demonstrated to promote progression of many types of solid cancer and induce about $20 \%$ of cancers [2]. Inflammation bowel disease (IBD) including Crohn's disease (CD) and ulcerative colitis (UC), which is the third highest risk factor of CRC [3], elevate the cancer incidence up to $20 \%$ [4]. About $2 \%$ cases of CRC arise in patients with long-standing IBD, known as colitis-associated cancer (CAC) [4].

The pathogenesis of CAC development is complex, and multiple factors including heredity, environmental factors, infection and imbalance of flora, modulate the microenvironment of IBD and induce immune disorders mediated by various immune cells and high level of pro-inflammatory cytokines, such as tumor necrosis factor-alpha (TNFa) and interleukin-6 (IL-6), which are responsible for the initiation and progression of CAC $[5,6]$. The duration and severity of inflammation in intestinal tissue are inextricably linked to neoplastic transformation of IBD [6]. TNFa, mainly induced by macrophages, can active multiple signal transduction, such as MAPK/JNK and nuclear factor kappa-B (NF-kB) pathways, contributing to cell survival, epithelial cell transformation and tumor progression [7].

Glucocorticoids (GCs) are used widely in the treatment of autoimmune and inflammatory diseases, due to their immunosuppressive and anti-inflammatory effects [8]. GCs are also used to treat lymphoid cancers and solid cancers associated symptoms or chemotherapy-related adverse reactions $[9,10]$. Moreover, GCs are effective to induce rapid remission of IBD, thus they are used as a mainstay of therapy, despite that their toxicity and side effects on various organs can not be neglected and the emerging immunosuppressive agents and antibodies have been used in the treatment of IBD [11]. However, the role of GCs in the initiation and progression of CAC is unclear.

The aim of this study is to explore the effects and underlying mechanisms of GCs in tumorigenesis and development of CAC. We first established the CAC murine model in C57BL/ 6 mice, induced by azoxymethane (AOM), a chemical carcinogen, followed with chronic colitis induced by three cycles of drinking water with dextran sodium sulfate (DSS) [12], which recapitulate the progression of CAC from normal mucosa to inflammation, hyperplasia and ultimately transforming into adenoma and carcinoma [13]. Based on the AOM/DSS-induced murine model, Dexamethasone (DEX), a synthetic analog of GCs, was given to mice at differential periods of the experiment, to determine therapeutic efficacy and molecular mechanisms of GCs in the development and progression of CAC.

\section{Materials And Methods}

\section{Reagents and antibodies}

Azoxymethane (AOM) and sodium dextran sulfate (DSS) were purchased from Sigma-Aldrich (St. Louis., MO, USA) and MP Biomedical (Carlsbad, CA, USA), respectively. Dexamethasone (DEX) was purchased from YEASEN Biotech Co. Ltd (Shanghai, China). The antibodies used in this study were presented in Table S1.

\section{Animal studies}


Male C57BL/ 6 mice ( $\mathrm{n}=80,8-9$ weeks) were purchased from the Laboratory Animal Center of Xi'an Jiaotong University (Xi'an, China), and housed in a specific-pathogen-free (SPF) condition under a $12 \mathrm{~h}$ light/dark cycle, with free access to a standard laboratory diet and water. All mice were adapted to above conditions for one week before the experiment, and 5-6 mice were housed in every cage.

All animal experiments were performed in compliance with Institution Guide-lines and approved by the Laboratory Animal Center of Xi'an Jiaotong University.

\section{Induction of CAC mouse model and glucocorticoids treatment}

We established the CAC mouse model as previously described [12]. Briefly, on the Day 1 of the experiment, the mice $(n=10)$ were injected intraperitoneally with $12 \mathrm{mg} / \mathrm{kg}$ of AOM dissolved in water and diluted with normal saline (NS) to a final concentration of $2 \mathrm{mg} / \mathrm{ml}$, and maintained with normal diet and water for one week. The mice were then given with drinking water containing $1.5 \% \mathrm{DSS}(\mathrm{wt} / \mathrm{vol})$ for 5 days, followed by 2 weeks of normal drinking water. This cycle was repeated for three times. After that, the mice recovered with regular diet and drinking water till the end of experiment. Control mice (Normal, $n$ =4) were injected intraperitoneally with $200 \mu$ normal saline (NS), and maintained with regular chow and drinking water till the end.

Next, the mice $(n=61)$ were induced CAC by AOM/DSS treatment. After the first DSS cycle, the mice $(n=$ 41) were divided in three groups: CAC $(n=11)$, DEX treatment at early stage (DEX-E, $n=18)$ and DEX treatment at late stage ( $D E X-L, n=12)$. The mice in CAC group were then injected intraperitoneally with $200 \mu$ normal saline (NS) containing 1\% DMSO after the second DSS cycle as negative control. Meanwhile, DEX ( $5 \mathrm{mg} / \mathrm{kg}$ ) dissolved in DMSO and diluted with normal saline (NS) were given intraperitoneally to the mice of DEX-E group every other day after the second cycle of DSS water, while the mice in DEX-L group were treated with DEX $(5 \mathrm{mg} / \mathrm{kg})$ after the last DSS cycle. Normal mice $(\mathrm{n}=4)$ were injected intraperitoneally with $200 \mu \mathrm{l}$ normal saline (NS) on the first day as control for AOM injection. All mice were monitored with body weight, stool consistency, rectal bleeding and survival ratio twice a week. Disease activity index (DAl) were scored by a semi-quantitative scoring system as previously described (Table S2) $[14,15]$.

At the end of the experiment (Day 95), all mice were sacrificed by cervical dislocation, and colon tissues and spleens were then collected. Besides, the fasting glucose level was measured before the mice sacrificed. For further analysis, the colons were cut open through longitudinal line, and washed with phosphate buffer saline (PBS, pH 7.4). Tumor number was then recorded and the diameter of each tumor was measured by an experimenter blindly (long diameter = length; short diameter = width; small tumor, medium tumor and large tumor are defined as the width $<2 \mathrm{~mm}, 2 \mathrm{~mm} \leq$ width $\leq 5 \mathrm{~mm}$, and $>5 \mathrm{~mm}$ ). Moreover, the area and volume of each tumor was calculated (tumor area $=\left[\left(\text { length }+ \text { width) }{ }^{\star} 0.5\right]^{2}\right.$, tumor volume $=$ length ${ }^{*}$ width ${ }^{2 *} 0.52$ ) as previously described [16]. Next, neoplastic tissues were fixed in $4 \%$ paraformaldehyde buffer immediately and then processed by dehydration, paraffin embedding and 
ultimately made into $5 \mu \mathrm{m}$ sections for histopathological examination (H\&E) and immunohistochemistry $(\mathrm{IHC})$ analysis, while the others were frozen in liquid nitrogen and stored at $-80{ }^{\circ} \mathrm{C}$ until use.

\section{Induction of colitis model and glucocorticoids treatment}

According to the above method, drinking water with $3 \%$ DSS was given to mice for 7 days.

To validate the anti-inflammatory effect of GCs, mice $(n=14)$ were divided into three groups: Normal $(n=$ $3)$, Colitis $(n=6)$ and Colitis-DEX $(n=5)$. The mice in Colitis-DEX group were injected intraperitoneally with DEX $(5 \mathrm{mg} / \mathrm{kg})$, while the mice in Colitis group were injected intraperitoneally with $200 \mu \mathrm{l}$ normal saline (NS) containing 1\% DMSO. During the experiment, mice were weighted every day, and stool consistency and rectal bleeding were recorded every other day. On Day 7, we tested the fasting glucose of all mice. Mice were then dissected, and colon and spleen tissues were fixed or stored at $-80^{\circ} \mathrm{C}$ until use.

\section{H\&E staining}

For histological evaluation of inflammation, paraffin-embedded colon sections were stained with H\&E, and the sections were then observed and evaluated blindly by a pathologist according to a previously established scoring system (Table S3) [17].

\section{Western blot analysis}

Colonic tissues were homogenized in RIPA buffer containing protease inhibitors, and equal amount of lysates were then subjected to western blot analysis as previously described [18]. All the experiments were performed in triplicate.

\section{Immunohistochemistry (IHC)}

The IHC staining was conducted to measure the levels of indicated proteins in colonic tissues. The experimental details were accomplished as previously described [18]. Each stained section was quantified using the Image $\mathrm{J}$, and scored with $0,1,2$ and 3, which represent negative, weak positive, positive, and strong positive, respectively. An average score was then used to evaluated the relative expression of proteins according to the staining intensity and percentage of positive area. Data shown are representative of independent experiments performed at least in triplicate.

\section{Enzyme-linked immunosorbent assay (ELISA)}

The tumors or colonic tissues were sampled and weighed, then homogenized in phosphate buffered saline (PBS). Next, the supernatant was collected and subjected to measure TNFa levels using the Mouse TNFa ELISA Kit (Excell Bio, Shanghai, China) according to the manufacturer's protocol. The values were normalized by tissue weight.

\section{Measurement of fasting blood glucose}

On the last day of the experiment, after deprivation of food for eight hours, the blood was taken from the tail of mice, and blood glucose was then measured with a glycometer (Sinocare, Changsha, China) according to the manufacturer's instruction. 


\section{Statistical analysis}

Student's unpaired $t$ test was performed to compare the means of data in two groups using GraphPad Prism Software Version 8.0 (GraphPad Software). All data were expressed as mean \pm standard deviation (SD), and $P$ values $<0.05$ were considered as a statistically significant difference.

\section{Results}

\section{The establishment of CAC mouse model}

AOM/DSS-induced colorectal cancer is a well-established, powerful and reproducible model in mice to study molecular pathogenesis of CAC [19]. First, we induced CAC in mice by AOM administration followed by repeated DSS cycle, separated by recovery period for two weeks. The detailed procedure was showed in Fig. 1a. During the experiment, body weight, rectal bleeding and stool consistency of mice were monitored twice a week. The results showed that AOM/DSS-treated mice showed significant loss of body weight compared to control mice at the period of DSS administration (Fig. 1b). At the end of experiment, the mice were sacrificed and dissected, and the colorectum was opened longitudinally for macroscopic observation. We tested a range of DSS concentrations previously, and found that CAC mice with $1.5 \%$ DSS treatment led to $100 \%$ incidence of multiple neoplasms in colons, particularly in the distal colon and rectum (Fig. 1C), with a mortality rate of about $40 \%$ (data not shown). There is no neoplasm in the colon of normal mice (Fig. 1C). According to pathologic evaluation on H\&E stained sections, most of them were adenoma with architectural complexities and completely lost of goblet cells (Fig. 1d).

\section{Dexamethasone treatment inhibits the development of colorectal carcinoma in AOM/DSS-induced mice}

Previous studies demonstrated that blocking inflammatory response has protective role in the development of CAC [20-22]. To explore whether GCs play an inhibitory role in colorectal tumorigenesis by its strong anti-inflammatory effect, we induced CAC in mice and administrated the mice with dexamethasone (DEX) after the second cycle of DSS (early intervention with DEX, DEX-E) or the third cycle (late intervention with DEX, DEX-L), as shown in Fig. 2a. Meanwhile, normal saline (NS) was given to model mice as control (CAC). Macroscopic view of the colon from each group was shown in Fig. $2 \mathrm{~b}$. DEX intervention significantly decreased tumor size, evidenced by the reduced number of large tumors (> $5 \mathrm{~mm}$ ) (Fig. 2c), but not smaller tumors ( $\leq 5 \mathrm{~mm}$ ), and decreased tumor area and tumor volume compared to those of the mice in CAC group (Fig. $2 \mathrm{~d}$ and e), although there was no significant reduction in the total number of colorectal tumors (Fig. 2f). Besides, our data showed that DEX treatment could not significantly reduce the mortality of AOM/DSS-induced mice due to the limited number of animals (Fig. 2g).

Next, we evaluated the expression of proliferation markers including cyclin D1 and Pcna by immunohistochemistry (IHC) assay. The results showed that the expression of cyclin D1 and Pcna was 
significantly elevated in tumor tissues from CAC mice compared to normal control, while their expression was strongly inhibited by DEX in both DEX-E and DEX-L groups (Fig. 3a and b). Taken together, our data indicate that DEX administration can inhibit the growth and progression of colorectal tumors in murine AOM/DSS-induced CAC model regardless of early or late intervention, in spite of the failure to prevent the incidence of tumors.

\section{DEX alleviates colonic inflammation in AOM/DSS-treated mice}

Inflammation mediated by multiple immune cells and cytokines plays an important role in promoting tumorigenesis and tumor progression [23]. TNFa shows an increased level in tumor microenvironment, and blocking TNFa signaling can decrease mucosal damage, infiltration of inflammatory cells and a significant reduction in tumor formation in AOM/DSS-treated mice [24]. Thus, we evaluated the inflammation and TNFa production of neoplasms and colonic tissues in AOM/DSS-treated mice. As shown in Fig. 4a and b, both western blot and IHC assays demonstrated an increased production of TNFa in tumor tissues of CAC mice compared to normal control, while TNFa production was reduced by DEX treatment. Notably, inhibitory effect was more prominent when DEX was administered at early stage. Besides, we detected local TNFa production in colon tissues by ELISA, and the results showed that DEX intervention decreased TNFa levels in neoplastic tissues, especially in DEX-E group (Fig. 4c).

To assess the infiltration of inflammatory cells in tumor tissues, H\&E staining was conducted, and the infiltration of inflammatory cells in neoplasm area was scored using a semi-quantitative method (Table S3). The results showed that the infiltration of inflammatory cells was significantly increased in tumor tissues of CAC mice compared to normal control, while reduced in DEX-treated mice compared to CAC mice regardless of the time of DEX intervention (Fig. $4 \mathrm{~d}$ ). Meanwhile, the expression of F4/80, a biomarker of mature macrophages, was also detected by $\mathrm{IHC}$ analysis. The results revealed that the infiltration of macrophages in tumor microenvironment was increased compared to normal control, while significantly decreased in DEX-treated mice (Fig. 4e). Besides, an abnormal increase in spleen size and weight was found in CAC mice compared to normal control, while DEX treatment induced clearly immunosuppression, evidenced by reduced size and weight of spleen compared to CAC mice, particularly in DEX-E group (Fig. 4f). Altogether, our data indicate that DEX intervention significantly alleviates colonic inflammation in AOM/DSS-treated mice.

\section{The effect of DEX intervention on the DSS-induced colitis}

To further determine the anti-inflammatory effect of DEX, we induced colitis in mice by $3 \%$ DSS administration for 7 days, and DEX ( $5 \mathrm{mg} / \mathrm{kg}$ ) was administered daily to mice in Colitis-DEX group from the second day (Fig. 5a). H\&E staining revealed that normal epithelial structure of colon disappeared and was infiltrated with inflammatory cells in DSS-treated mice, while DEX administration markedly inhibited the infiltration of inflammatory cells, and the retained mucosal structure of intestine could be observed in Colitis-DEX mice (Fig. 5b). Meanwhile, intestinal inflammation was histologically evaluated on the basis 
of four parameters: inflammatory infiltration, mucosal injury, crypt damage and lesion area (Table S3). The results demonstrated that inflammatory score of DEX-treated mice was lower than that in colitis mice (Fig. 5c), indicating that DEX protected mice against DSS-induced colitis in colon. This was consistent with the results that DEX-treated mice had lower weight/length ratio compared to colitis mice (Supplementary Figure S1). As supported, F4/80 staining showed that macrophage infiltration was significantly elevated in colitis mice compared to normal control, while DEX intervention effectively revised this effect (Fig. 5d).

Next, we used ELISA to measure TNFa levels in colonic tissues, and found increased levels of TNFa in colonic tissues of colitis mice compared to normal control, while DEX treatment could reverse this effect (Fig. 5e). This was also supported by the results of western blot and IHC assays (Fig. $5 f$ and g). As expected, we found that the spleen weight was increased in colitis mice compared to normal control, although the difference did not reach statistical significance, while DEX treatment effectively reduced the spleen weight compared to colitis mice (Fig. 5h). Besides, we attempted to determine the effect of DEX intervention on the expression of cyclin D1 and Pcna in colitis mice. The results showed that the expression of cyclin D1 and Pcna was elevated in colonic tissues of colitis mice compared to normal control, but there was no significant difference between them, while DEX treatment effectively downregulated their expression (Fig. 6a). This was also supported by the results of IHC assays (Fig. 6b and c). Collectively, our data indicate that DEX not only plays an anti-inflammatory effect in DSS-induced colitis, but also inhibits the expression of proliferative markers in colonic tissues.

\section{DEX treatment inhibits the activity of MAPK/JNK pathway}

The MAPK pathway including JNK, which is activated by inflammatory mediators such as TNFa, is often dysregulated in inflammation response and tumorigenesis [25]. Our above studies have demonstrated that DEX intervention can decrease TNFa production in CAC or colitis mouse model. Thus, we speculate that DEX treatment inhibits the activity of JNK pathway by decreasing TNFa production. The results showed that the levels of total or phosphorylated Jnk (p-Jnk) were increased in AOM/DSS-induced mice compared to normal control, while their levels were clearly decreased by DEX, particularly early DEX intervention (Fig. 7a), also supported by the results of IHC assays (Fig. 7b). Besides, we also demonstrated the inhibitory effect of DEX intervention on the activity of JNK pathway in DSS-induced colitis by western blot and IHC assays (Fig. 7c and d). These observations indicate that DEX exerts its anti-inflammatory and anti-tumor properties in DSS-induced colitis and colitis-associated cancer at least partially by inhibiting the activity of MAPK/JNK pathway.

\section{DEX treatment induces general deterioration of mouse state}

The duration of glucocorticoids therapy results in various side effects, such as peptic ulcer, osteoporosis and immunosuppression, limiting their application to a certain extent [26]. A previous study based on DSS-induced colitis model has revealed that budesonide aggravates body weight loss, rectal bleeding and impaired epithelium healing [27]. In this study, we recorded body weight, rectal bleeding and stool 
consistency to monitor the general state of mouse. For AOM/DSS-treated mice, body weight significantly decreased in the period of DSS administration compared to normal control (Fig. 8a). Moreover, DEX treatment resulted in greater loss of body weight at the end of experiment (Fig. 8b). Similarly, a sustained and aggravated reduction of body weight was observed in DEX-treated mice compared to the mice in colitis model (Fig. 8c and d).

We also evaluated the disease severity of DSS administration, based on disease activity index (DAI) scoring system, including body weight loss, rectal bleeding and stool consistency (Table S2). Throughout the DSS-treated procedure, we found that DEX administration was associated with exacerbated severity of body weight loss, rectal bleeding and diarrhea. As shown in Fig. 8e, DAl score increased and reached the highest levels in every cycle of DSS administration, and a higher DAI score was observed after the second DSS cycle in DEX-treated mice. Similar results were also found in DSS-induced colitis (Fig. 8f). Besides, we observed that majority of mice in DEX-treated group became weaker manifested in anorexia and reduced activity (data not shown). Thus, we tested the fasting blood-glucose of all mice at the last day of the experiment. Expectedly, DEX intervention decreased the fasting glucose levels in CAC mice regardless of the time of DEX administration (Fig. 8g). Similarly, DEX induced a lower level of blood glucose in mice suffered from DSS-induced colitis (Fig. 8h). These data suggest that DEX treatment attenuates the tolerance of mice to DSS challenge and induced general deterioration of mouse statue.

\section{Discussion}

It is clear that chronic inflammation is recognized as a promoting factor in tumor initiation and development including colorectal cancer[2]. The patients suffering from IBD have an increased risk of developing to colorectal cancer, in which the longstanding of colonic inflammation may play a pathogenic role in malignant transformation [6]. Although the etiology and pathology of IBD have not been defined completely, which has been recently ascribed to impaired barrier function and immune disorder resulting from the complexity genetic background and various defined or undefined environmental factors [28, 29]. It is well-established that blocking cytokines-mediated inflammatory pathways can reduce tumorigenesis of CAC, supported by several discoveries. For example, blocking TNFa pathway and IL- 6 deficiency reduced tumor formation in mice treated by AOM and DSS $[22,30]$. TcES treatment inhibited AOM/DSS-induced tumorigenesis by regulating both STAT3 and NF-KB signaling pathways [20]. The application of GCs in therapy of IBD has not diminished, despite of the toxicity and increased risk of infection, due to long-term administration [6, 11]. A growing number of studies have investigated the role of GCs in tumor development and revealed their distinct mechanisms that promote or inhibit cancer progression in different types of cancer, indicating that their activity was not limited to anti-inflammation [31-35]. Thus, it is impending to explore the effect and underlying mechanism of GCs in colitis-associated cancer.

In this study, DEX was administered after the second or the third cycle of DSS drinking, which simulated the fact that GCs were given to induce rapid remission after the symptoms of IBD were observed. We concluded that DEX played an anti-inflammatory role in experimental colitis in mice, evidenced by 
decreased histological score, releasing of TNFa, and infiltration of mature macrophagocytes. We also observed that DEX aggravated body weight loss, rectal bleeding and elevated DAI score throughout of the experiment. As supported, a previous study revealed that budesonide had both protective and deleterious effects in DSS-induced colitis, and demonstrated that budesonide impaired wound healing and weakened mucosal barrier function by inhibiting epithelial proliferation, evidenced by decreased expression of claudin-2, PCNA, cyclin D1, cytokeratin 5/8 and claudin-4 in budesonide treated mice [27]. Similarly, another study analyzed and compared the efficiency of key compounds used in the treatment of IBD, implying that budesonide, one of the synthetic glucocorticoids, had limited activity in preventing DSSinduced colitis due to its severe side effects [36]. This will mask its inhibitory effect on local inflammation and induce unfavorable outcome.

The immune cells and inflammatory mediators in the tumor microenvironment play a crucial role in tumor promotion [37]. Inflammatory response and tumorigenesis are frequently associated with dysregulation of MAPK pathways, including JNK, which is activated by pro-inflammatory cytokines [38], and has been demonstrated to take part in the survival and proliferation of tumor cells [25]. Of them, TNFa plays a critical role in inflammatory response and tumor progression by targeting both immune and tumor cells and activating various oncogenic pathways, including MAPK/JNK-AP1 pathway [39]. A previous study has demonstrated that JNK in macrophages play a critical role in inflammatory response and development of hepatocellular carcinoma using myeloid cell-specific JNK-deficient mice [40]. In the present study, we found increased staining score of $p$-Jnk in stromal tissues of mice with CAC or colitis, and DEX administration could alleviate the inflammation and inhibit the growth of tumors in colonic tissue in AOM/DSS-induced CAC mouse model by blocking the MAPK/JNK pathway. This was supported by a previous study that DEX administration decreased the content of JNK protein and phosphorylated JNK [41].

The fact that DEX administration from both the second cycle and the third cycle of DSS drinking significantly reduced tumor size in colon, while had no effect on total tumor number, suggesting that the first cycle of DSS administration may be critical for AOM/DSS-induced tumorigenesis. Besides, although DEX intervention at early stage showed greater suppression of colonic inflammation, the inhibitory effect on tumor progression was just as prominent as that in DEX intervention at late stage. This was at least partly ascribed to the destruction of tumor immunology due to the long-term immunosuppression by DEX administration. As supported, previous studies demonstrated that DEX could suppress the activation of T cell by inhibiting inflammatory cytokines and upregulating PD-1 expression [42, 43]. There is evidence showing that DEX administration can impair intratumoral immunity, thereby causing the failure of cancer immunotherapy in vivo [44]. These observations indicate that immunosuppression and modulation of tumor microenvironment by GCs exposure may contribute to tumor progression [45].

Recently, there was study demonstrating that DEX accelerated the onset and severity of colitis and colitisassociated cancer mediated by mTOR signaling pathway, in which mice were treated with DEX (2.5 $\mathrm{mg} / \mathrm{kg}$ ) at the beginning of DSS drinking [46]. Besides, another study concluded that corticosterone, which was given in feeding solution ( $50 \mathrm{mg} / \mathrm{L}$ ) one week after DSS administration, could inhibit the 
inflammation but promoted the development of AOM/DSS-induced colon cancer by activating NF-KB and COX-2 [16]. This may be explained by that different options and periods of drug feeding will cause diverse effects on experimental animals. These studies suggest that GCs have different effects on tumor progression and inflammatory response, depending on the form and dosage of GCs, time and duration of drug treatment [9], predisposition of animal, the concentration and duration of DSS administration [36]. High dose and high frequency of GCs may be necessary to obtain their anti-tumor activity [47], but the prior exposure of DEX may be unfavorable, even stimulate inflammatory response [48]. Thus, to define proper dosage and time window of GCs application, robust pre-clinical studies and clinical trials are desired to carry out in the near future.

In this study, we systematically determined the different effects of GCs given at different points of time on colitis-associated tumorigenesis and tumor progression. Our results implied that DEX intervention suppressed DSS-induced colitis and the progression of AOM/DSS-induced CAC in mice. However, DEX treatment aggravated the body weight loss and rectal bleeding in mice with DSS exposure. These findings indicate that the strict selection of time and dosage in GCs treatment become crucially important whether in experimental studies or clinical practices, to gain their inhibitory activities and reduce the side effects. Thus, we need to enlarge the number of animals and subdivide groups to further clarify the different effects of GCs under different conditions. Besides, prospective clinical trials are needed to assess the effect of GCs in the progression of colitis-associated cancer.

\section{Conclusion}

In summary, our data demonstrate that DEX has an anti-inflammatory effect on DSS-induced colitis and inhibits tumor progression in AOM/DSS-induced CAC, at least partially associated with the inhibition of MAPK/JNK pathway. These results support the therapeutic potential of GCs in colitis-associated cancer, while its deleterious effect on body weight loss and rectal bleeding may limit the duration of GCs administration.

\section{Abbreviations}

GCs, Glucocorticoids; IBD, inflammatory bowel disease; CAC, colitis-associated cancer; AOM, azoxymethane; DSS, dextran sodium sulphate; DEX, Dexamethasone; PCNA, proliferating cell nuclear antigen; TNFa, tumor necrosis factor a; MAPK, Mitogen-activated protein kinase; JNK, c-Jun N-terminal kinase; CRC, colorectal cancer; CD, Crohn's disease; UC, ulcerative colitis; DEX-E, DEX treatment at early stage; DEX-L, DEX treatment at late stage; NS, normal saline; DMSO, dimethylsulfoxide; DAl, Disease activity index; H\&E, hematoxylin and eosin; IHC, immunohistochemistry; ELISA, Enzyme-linked immunosorbent assay; STAT3, signal transducer and activator of transcription 3; NF-kB, nuclear factor kappa-light-chain-enhancer of activated B cells.

\section{Declarations}




\section{Ethics approval and consent to participate}

All animals' experimental procedures were performed in compliance with Institution Guide-lines and approved by Institutional Review Board of Xi'an Jiaotong University.

\section{Consent for publication}

Not applicable.

\section{Availability of data and materials}

All data generated or analyzed during this study are included in this article.

\section{Competing interests}

The authors declare that they have no competing interest.

\section{Funding}

This work was supported by the National Natural Science Foundation of China (No. 81972593 to M.J.), Innovation Talent Promotion Plan in Shaanxi Province (No. 2018TD-006 to P.H.) and the Science and Technology Project of Shaanxi Province (No. 2019SF-015 to P.H.).

\section{Authors' contributions}

M.J. conceived and designed the experiments. J.P., X.Z. and J.L. performed the experiments and analyzed the data. P.H. and M.J. contributed reagents and materials. M.J. and P.H. wrote the manuscript. All authors read and approved the final manuscript.

\section{Acknowledgements}

Not applicable.

\section{References}

1. Sung H, Ferlay J, Siegel RL, Laversanne M, Soerjomataram I, Jemal A, et al. Global cancer statistics 2020: GLOBOCAN estimates of incidence and mortality worldwide for 36 cancers in 185 countries. CA Cancer J Clin. 2021;0:1-41.

2. Galdiero MR, Marone G, Mantovani A. Cancer Inflammation and Cytokines. Cold Spring Harb Perspect Biol. 2018;10(8):a028662.

3. Kim ER, Chang DK. Colorectal cancer in inflammatory bowel disease: the risk, pathogenesis, prevention and diagnosis. World J Gastroenterol. 2014;20(29):9872-81.

4. Francescone R, Hou V, Grivennikov SI. Cytokines, IBD, and colitis-associated cancer. Inflamm Bowel Dis. 2015;21(2):409-18. 
5. Chen L, Zhou Z, Yang Y, Chen N, Xiang H. Therapeutic effect of imiquimod on dextran sulfate sodium-induced ulcerative colitis in mice. PLoS One. 2017;12(10):e0186138.

6. Saraggi D, Fassan M, Mescoli C, Scarpa M, Valeri N, Michielan A, et al. The molecular landscape of colitis-associated carcinogenesis. Dig Liver Dis. 2017;49(4):326-30.

7. Bertazza L, Mocellin S. The Dual Role of Tumor Necrosis Factor (TNF) in Cancer Biology. Curr Med Chem. 2010;17(29):3337-52.

8. Spies CM, Strehl C, van der Goes MC, Bijlsma JW, Buttgereit F. Glucocorticoids Best Pract Res Clin Rheumatol. 2011;25(6):891-900.

9. Lin KT, Wang LH. New dimension of glucocorticoids in cancer treatment. Steroids. 2016;111:84-8.

10. Pufall MA. Glucocorticoids and Cancer. Adv Exp Med Biol. 2015;872:315-33.

11. Bernstein CN. Treatment of IBD: where we are and where we are going. Am J Gastroenterol. 2015;110(1):114-26.

12. Neufert $C$, Becker $C$, Neurath MF. An inducible mouse model of colon carcinogenesis for the analysis of sporadic and inflammation-driven tumor progression. Nat Protoc. 2007;2(8):1998-2004.

13. Snider AJ, Bialkowska AB, Ghaleb AM, Yang VW, Obeid LM, Hannun YA. Murine Model for ColitisAssociated Cancer of the Colon. Methods Mol Biol. 2016;1438:245-54.

14. Coronado S, Barrios L, Zakzuk J, Regino R, Ahumada V, Franco L, et al. A recombinant cystatin from Ascaris lumbricoides attenuates inflammation of DSS-induced colitis. Parasite Immunol. 2017;39(4):e12425.

15. Zhang Z, Shen P, Xie W, Cao H, Liu J, Cao Y, et al. Pingwei San ameliorates dextran sulfate sodiuminduced chronic colitis in mice. J Ethnopharmacol. 2019;236:91-9.

16. Li B, Wang Y, Yin L, Huang G, Xu Y, Su J, et al. Glucocorticoids promote the development of azoxymethane and dextran sulfate sodium-induced colorectal carcinoma in mice. BMC Cancer. 2019;19(1):94.

17. Gao Z, Yu C, Liang H, Wang X, Liu Y, Li X, et al. Andrographolide derivative CX-10 ameliorates dextran sulphate sodium-induced ulcerative colitis in mice: Involvement of NF-kappaB and MAPK signalling pathways. Int Immunopharmacol. 2018;57:82-90.

18. Wang N, Li Y, Wei J, Pu J, Liu R, Yang Q, et al. TBX1 Functions as a Tumor Suppressor in Thyroid Cancer Through Inhibiting the Activities of the PI3K/AKT and MAPK/ERK Pathways. Thyroid. 2019;29(3):378-94.

19. Parang B, Barrett CW, Williams CS. AOM/DSS Model of Colitis-Associated Cancer. Methods Mol Biol. 2016;1422:297-307.

20. Callejas BE, Mendoza-Rodriguez MG, Villamar-Cruz O, Reyes-Martinez S, Sanchez-Barrera CA, Rodriguez-Sosa $\mathrm{M}$, et al. Helminth-derived molecules inhibit colitis-associated colon cancer development through NF-kappaB and STAT3 regulation. Int J Cancer. 2019;145(11):3126-39.

21. Li ZW, Sun B, Gong T, Guo S, Zhang J, Wang J, et al. GNAl1 and GNAl3 Reduce Colitis-Associated Tumorigenesis in Mice by Blocking IL6 Signaling and Down-regulating Expression of GNAI2. 
Gastroenterology. 2019;156(8):2297-312.

22. Popivanova BK, Kitamura K, Wu Y, Kondo T, Kagaya T, Kaneko S, et al. Blocking TNF-alpha in mice reduces colorectal carcinogenesis associated with chronic colitis. J Clin Invest. 2008;118(2):560-70.

23. Wang J, Li D, Cang H, Guo B. Crosstalk between cancer and immune cells: Role of tumor-associated macrophages in the tumor microenvironment. Cancer Med. 2019;8(10):4709-21.

24. De Robertis M, Massi E, Poeta ML, Carotti S, Morini S, Cecchetelli L, et al. The AOM/DSS murine model for the study of colon carcinogenesis: From pathways to diagnosis and therapy studies. $J$ Carcinog. 2011;10:9.

25. Wagner EF, Nebreda AR. Signal integration by JNK and p38 MAPK pathways in cancer development. Nat Rev Cancer. 2009;9(8):537-49.

26. Oray M, Abu Samra K, Ebrahimiadib N, Meese H, Foster CS. Long-term side effects of glucocorticoids. Expert Opin Drug Saf. 2016;15(4):457-65.

27. Ocon B, Aranda CJ, Gamez-Belmonte R, Suarez MD, Zarzuelo A, Martinez-Augustin O, et al. The glucocorticoid budesonide has protective and deleterious effects in experimental colitis in mice. Biochem Pharmacol. 2016;116:73-88.

28. Choi CR, Bakir IA, Hart AL, Graham TA. Clonal evolution of colorectal cancer in IBD. Nat Rev Gastroenterol Hepatol. 2017;14(4):218-29.

29. Neurath MF. Cytokines in inflammatory bowel disease. Nat Rev Immunol. 2014;14(5):329-42.

30. Grivennikov S, Karin E, Terzic J, Mucida D, Yu GY, Vallabhapurapu S, et al. IL-6 and Stat3 are required for survival of intestinal epithelial cells and development of colitis-associated cancer. Cancer Cell. 2009;15(2):103-13.

31. Han S, Bui NT, Ho MT, Kim YM, Cho M, Shin DB. Dexamethasone Inhibits TGF-beta1-Induced Cell Migration by Regulating the ERK and AKT Pathways in Human Colon Cancer Cells Via CYR61. Cancer Res Treat. 2016;48(3):1141-53.

32. Kim JH, Hwang YJ, Han SH, Lee YE, Kim S, Kim YJ, et al. Dexamethasone inhibits hypoxia-induced epithelial-mesenchymal transition in colon cancer. World J Gastroenterol. 2015;21(34):9887-99.

33. Lin KT, Yeh YM, Chuang CM, Yang SY, Chang JW, Sun SP, et al. Glucocorticoids mediate induction of microRNA-708 to suppress ovarian cancer metastasis through targeting Rap1B. Nat Commun. 2015;6:5917.

34. Sorrentino G, Ruggeri N, Zannini A, Ingallina E, Bertolio R, Marotta C, et al. Glucocorticoid receptor signalling activates YAP in breast cancer. Nat Commun. 2017;8:14073.

35. Wu Y, Xia R, Dai C, Yan S, Xie T, Liu B, et al. Dexamethasone inhibits the proliferation of tumor cells. Cancer Manag Res. 2019;11:1141-54.

36. Sann H, Erichsen J, Hessmann M, Pahl A, Hoffmeyer A. Efficacy of drugs used in the treatment of IBD and combinations thereof in acute DSS-induced colitis in mice. Life Sci. 2013;92(12):708-18.

37. Danese S, Malesci A, Vetrano S. Colitis-associated cancer: the dark side of inflammatory bowel disease. Gut. 2011;60(12):1609-10. 
38. Hammouda MB, Ford AE, Liu Y, Zhang JY. The JNK Signaling Pathway in Inflammatory Skin Disorders and Cancer. Cells. 2020;9(4):857.

39. Taniguchi K, Karin M. NF-kappaB, inflammation, immunity and cancer: coming of age. Nat Rev Immunol. 2018;18(5):309-24.

40. Han MS, Barrett T, Brehm MA, Davis RJ. Inflammation Mediated by JNK in Myeloid Cells Promotes the Development of Hepatitis and Hepatocellular Carcinoma. Cell Rep. 2016;15(1):19-26.

41. Motta K, Barbosa AM, Bobinski F, Boschero AC, Rafacho A. JNK and IKKbeta phosphorylation is reduced by glucocorticoids in adipose tissue from insulin-resistant rats. J Steroid Biochem Mol Biol. 2015;145:1-12.

42. Maeda N, Maruhashi T, Sugiura D, Shimizu K, Okazaki IM, Okazaki T. Glucocorticoids potentiate the inhibitory capacity of programmed cell death 1 by up-regulating its expression on $\mathrm{T}$ cells. J Biol Chem. 2019;294(52):19896-906.

43. Xing K, Gu B, Zhang P, Wu X. Dexamethasone enhances programmed cell death 1 (PD-1) expression during $T$ cell activation: an insight into the optimum application of glucocorticoids in anti-cancer therapy. BMC Immunol. 2015;16:39.

44. Flint TR, Janowitz T, Connell CM, Roberts EW, Denton AE, Coll AP, et al. Tumor-Induced IL-6 Reprograms Host Metabolism to Suppress Anti-tumor Immunity. Cell Metab. 2016;24(5):672-84.

45. Volden PA, Conzen SD.. .; Suppl. The influence of glucocorticoid signaling on tumor progression. Brain Behav Immun. 2013;30:S26-31.

46. Zhang Z, Dong L, Jia A, Chen X, Yang Q, Wang Y, et al. Glucocorticoids Promote the Onset of Acute Experimental Colitis and Cancer by Upregulating mTOR Signaling in Intestinal Epithelial Cells. Cancers. 2020;12(4):945.

47. Banciu M, Schiffelers RM, Metselaar JM, Storm G. Utility of targeted glucocorticoids in cancer therapy. J Liposome Res. 2008;18(1):47-57.

48. Frank MG, Miguel ZD, Watkins LR, Maier SF. Prior exposure to glucocorticoids sensitizes the neuroinflammatory and peripheral inflammatory responses to E. coli lipopolysaccharide. Brain Behav Immun. 2010;24(1):19-30.

\section{Figures}


a

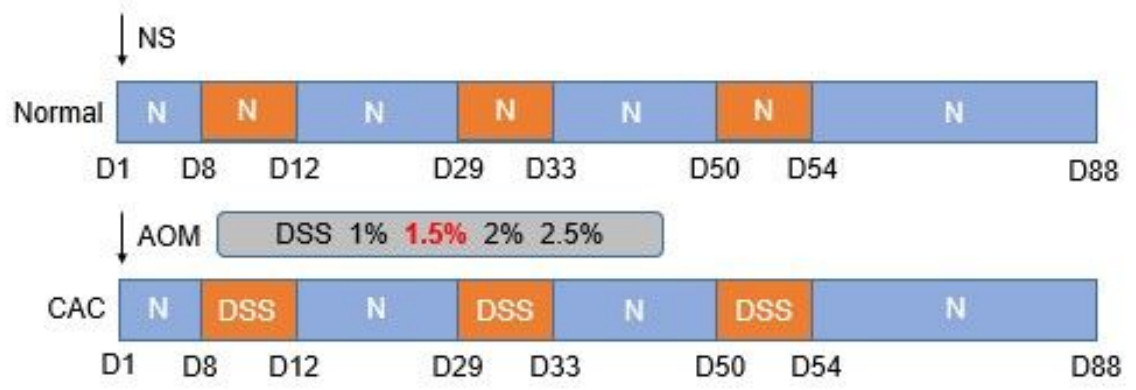

b

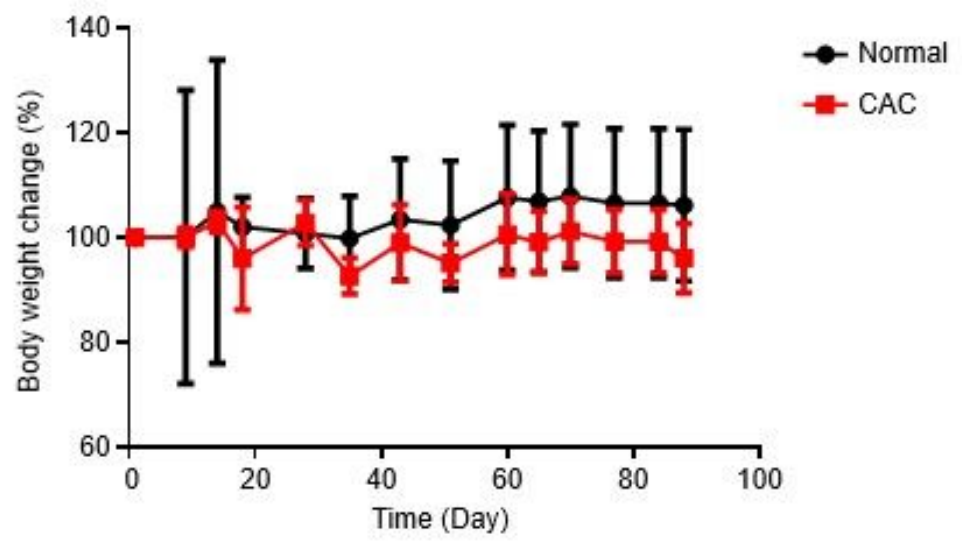

C

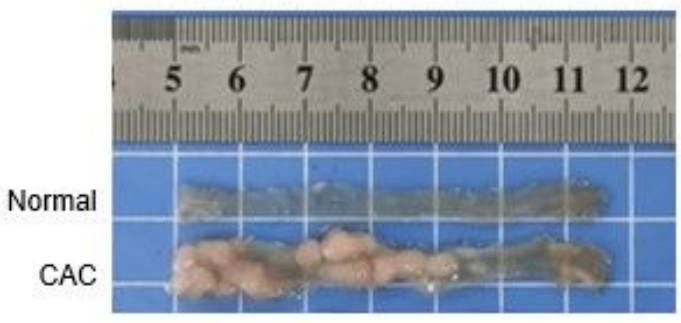

d

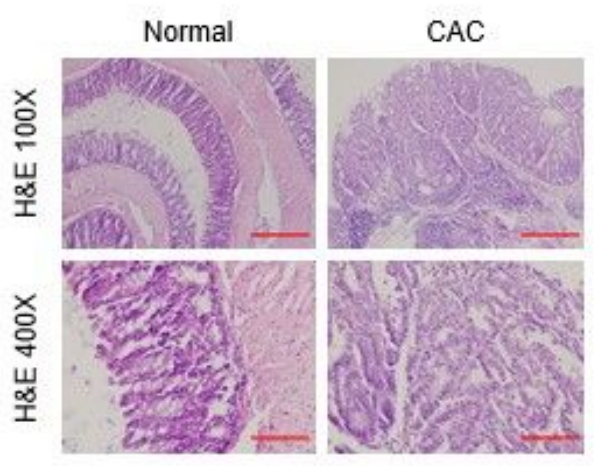

\section{Figure 1}

The establishment of CAC mouse model. a, The scheme shows the process of AOM/DSS-induced colitis associated cancer (CAC). A single-dose of AOM $(12 \mathrm{mg} / \mathrm{kg}$ ) was injected to mice followed with three cycles of drinking water with series of concentrations of DSS for 5 days and recovery period for 2 weeks. The tumors in colon was examined about one month after the third DSS cycle. b, Body weight change in normal mice $(n=4)$ and AOM/DSS-treated mice $(n=6)$. c, The representative view of neoplasms in colon of AOM/DSS-treated mice, and the colon of normal mice was presented as control. $d$, Histological views of neoplasms in AOM/DSS-treated mice and normal epithelium (H\&E staining); 100x amplification in upper panels and 400x amplification in lower panels; the scale represents $100 \mu \mathrm{m}$. Data were expressed as mean \pm SD; AOM, azoxymethane; DSS, dextran sodium sulphate; NS, normal saline; CAC, colitis associated cancer; $H \& E$, hematoxylin and eosin. 
a

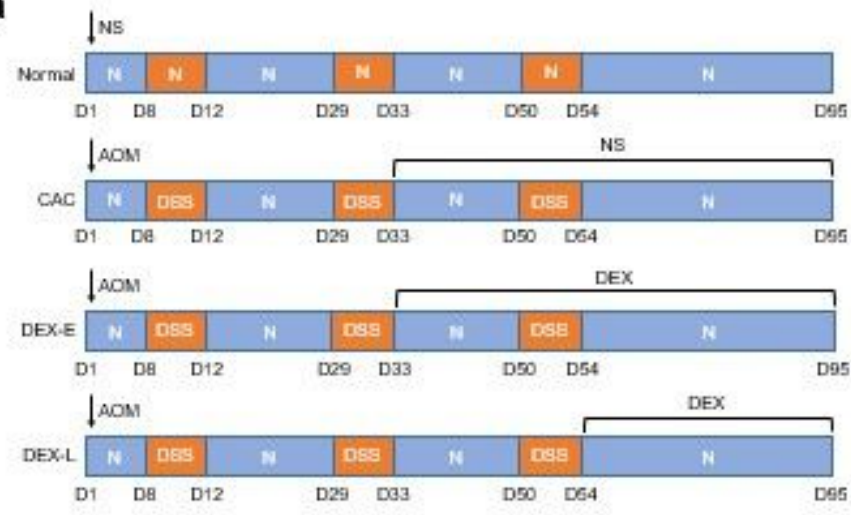

b

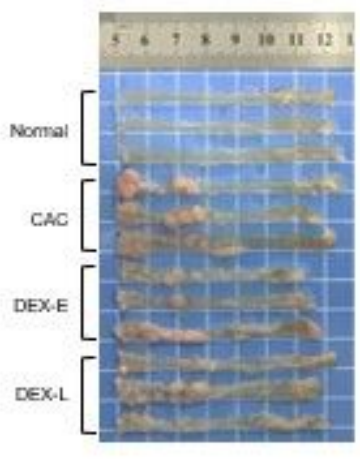

c
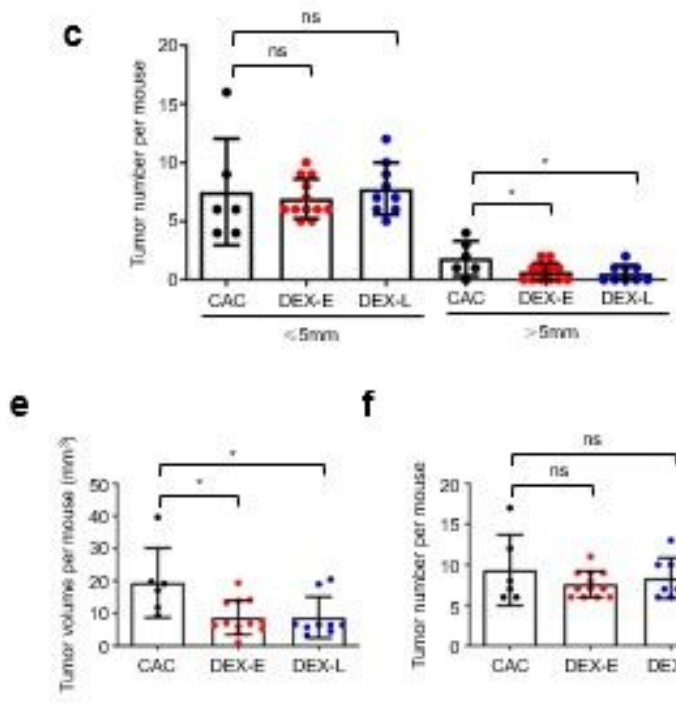

d

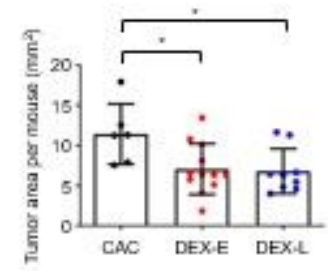

g

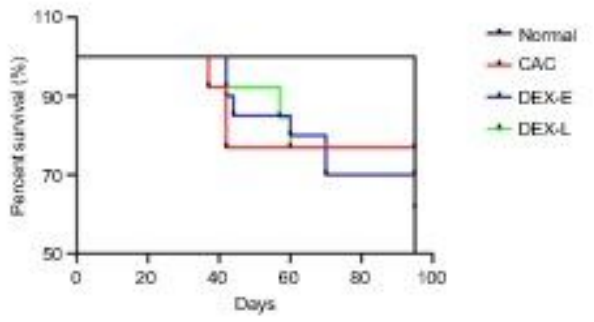

\section{Figure 2}

DEX treatment inhibits tumor growth in CAC model. a, AOM/DSS-treated mice were divided into different groups. The normal mice (Normal, $n=4$ ) were injected intraperitoneally with $200 \mu$ normal saline (NS) as the controls for AOM injection. The AOM/DSS-induced mice were treated with NS containing 1\% DMSO (CAC, $n=11$ ) or subjected to DEX intervention ( $5 \mathrm{mg} / \mathrm{kg}$, ip) after the second cycle of DSS (DEX-E, $n=18$ ) or the third cycle of DSS (DEX-L, $n=12$ ). The tumors in colon were measured at $D 95$. $b$, The representative longitudinal views of coloretum of mice in different groups ( $n=3$ per group). c, Comparison of the numbers of small to mediate tumors (diameter $\leq 5 \mathrm{~mm}$ ) and large tumors (diameter $>5 \mathrm{~mm}$ ) among different groups. $d$, Comparison of tumor area among different groups. e, Comparison of tumor volume among different groups. $\mathrm{f}$, Comparison of total number of tumors among different groups. $\mathrm{g}$, Comparison of survival rate of mice among different groups. The number of mice in Normal, CAC, DEX-E and DEX-L groups was 4, 6, 12 and 9, respectively. All data are expressed as mean $\pm S D$. ${ }^{*}, P<0.05$; ns, not significant. 
a

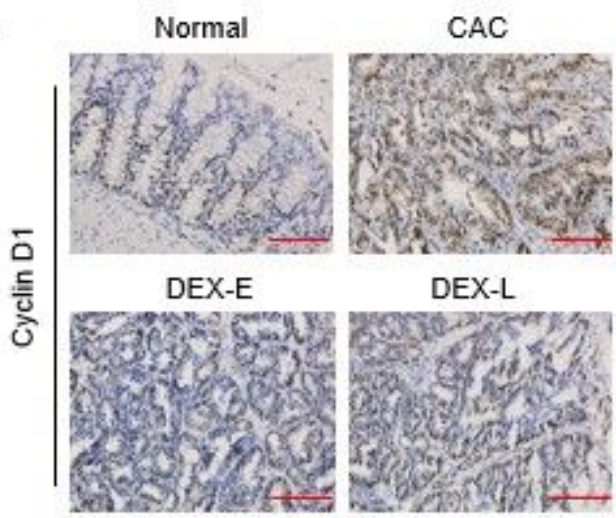

b

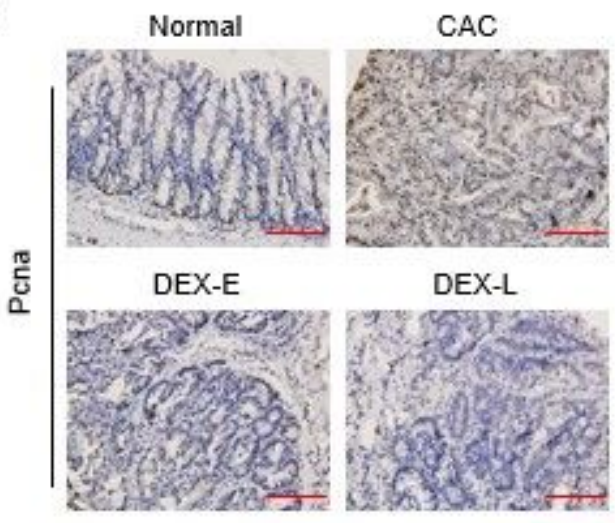

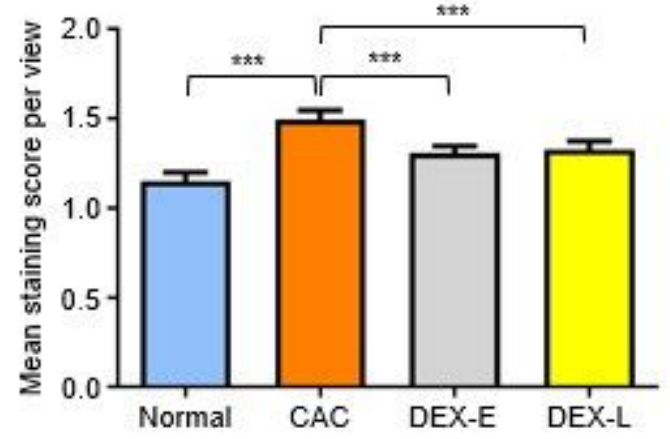

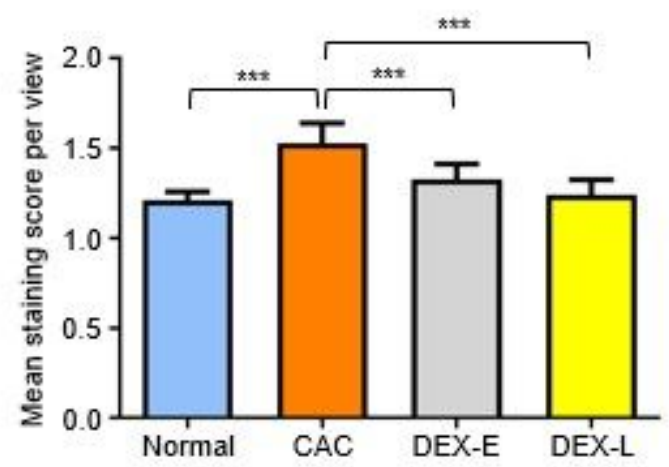

Figure 3

DEX treatment inhibits the expression of cyclin D1 and Pcna. The IHC assay was performed to evaluate the expression of proliferative markers cyclin D1 and Pcna in neoplastic tissues or normal colonic tissues of mice in different groups. Left panels show the representative images (400x) of IHC staining of cyclin D1 (a) and Pcna (b) in colonic epitheliums in the indicated mice ( $\mathrm{n}=3$ per group), and statistical results were presented in right panels. The scale represents $100 \mu \mathrm{m}$. Data were expressed as mean \pm SD. $\star \star \star, P<$ 0.001 . 


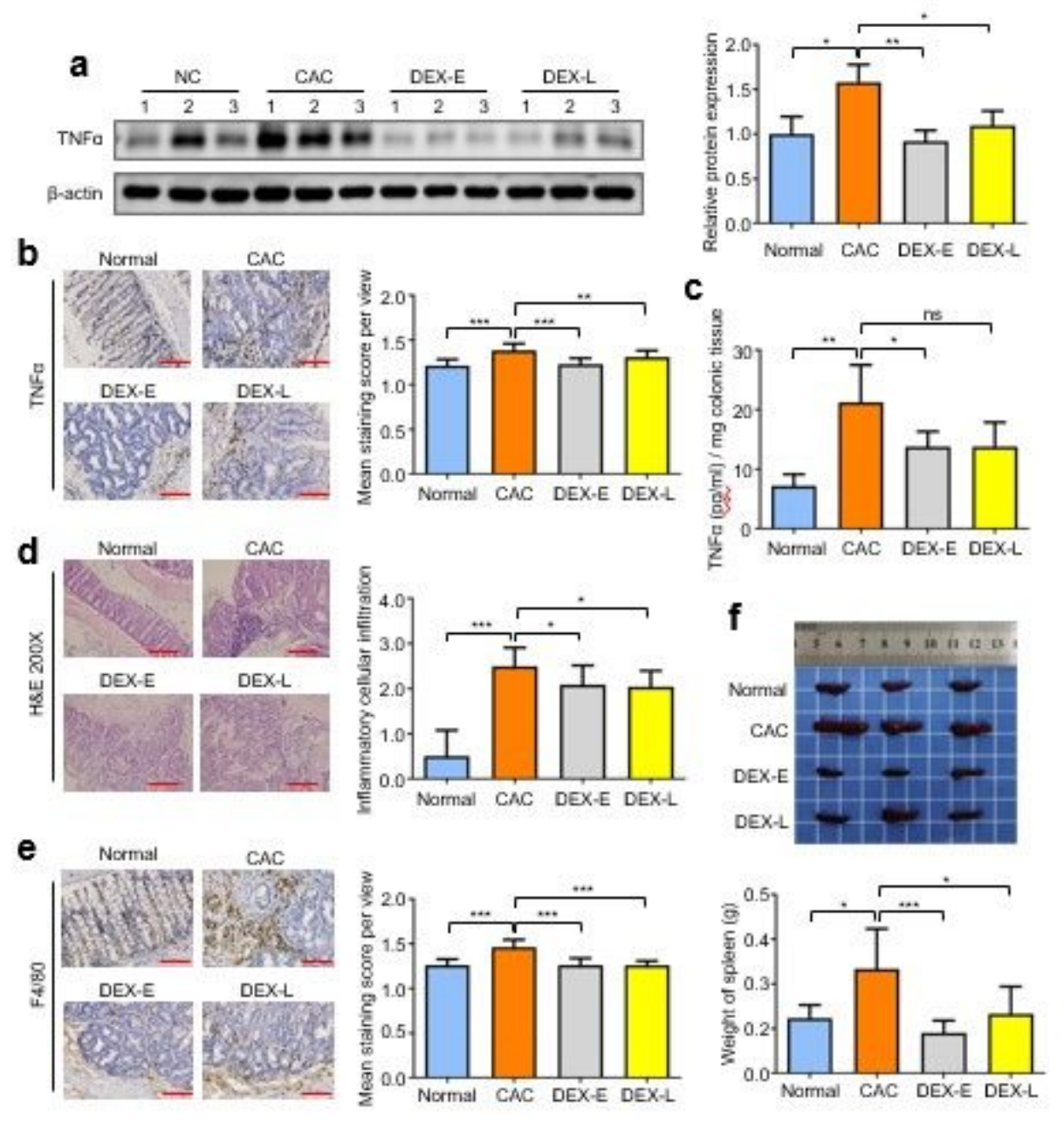

\section{Figure 4}

DEX treatment inhibits the colonic inflammation in AOM/DSS-induced mice. DEX ( $5 \mathrm{mg} / \mathrm{kg}$, ip) was given to AOM/DSS-treated mice at different points of time to determine its anti-inflammatory effect. a, TNFa expression was detected by western blot analysis (left panel, $n=3$ per group) and measured using densitometry. $\beta$-action was used as a loading control. Statistical results were presented in right panel. b, Left panels show the representative IHC images of TNFa staining in colonic epitheliums of normal mice and neoplastic tissues of the indicated mice ( $n=3$ per group), and statistical results were presented in right panel. c, TNFa production in colonic tissues was detected by ELISA and normalized by the weight of tissue specimens in Normal $(n=3), \operatorname{CAC}(n=6)$, DEX-E $(n=8)$ and DEX-L $(n=7)$ mice. $d$, Left panels show the representative images of normal epithelium and neoplasm area of AOM/DSS-treated mice (H\&E staining, 200x amplification), and local infiltration of inflammatory cells was scored by a semiquantitative method (right panel). e, The infiltration of macrophages in tumors was measured by IHC assay with $F 4 / 80$ antibody (left panels, $n=3$ per group). Statistical results were presented in right panel. $f$, The spleen images (upper panel) and statistical results of spleen weight (lower panel) in the indicated mice $\left(n=3\right.$ per group). The scale represents $100 \mu \mathrm{m}$. All data were expressed as mean \pm SD. ${ }^{*}, P<0.05$; $* *$, $\mathrm{P}<0.01$; $* \star \star, P<0.001 ;$ ns, not significant. 
a

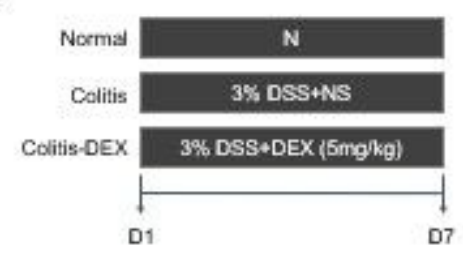

b

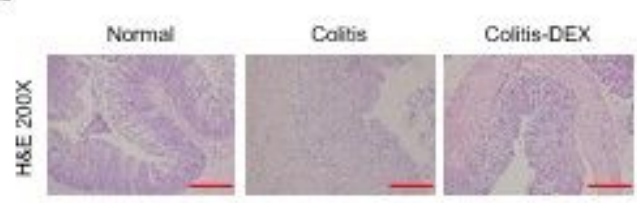

C

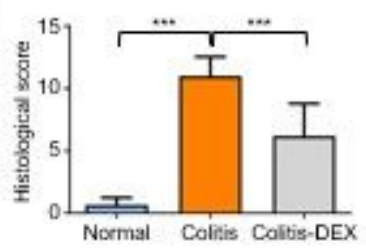

d

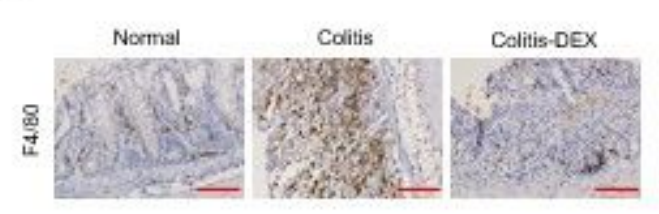

$\mathbf{f}$

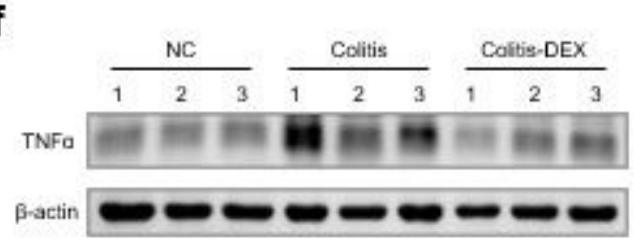

g

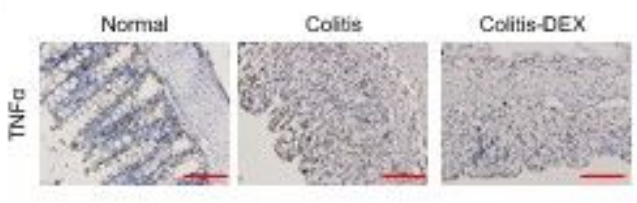

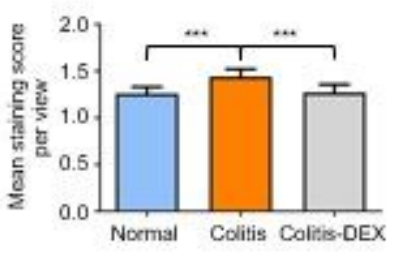
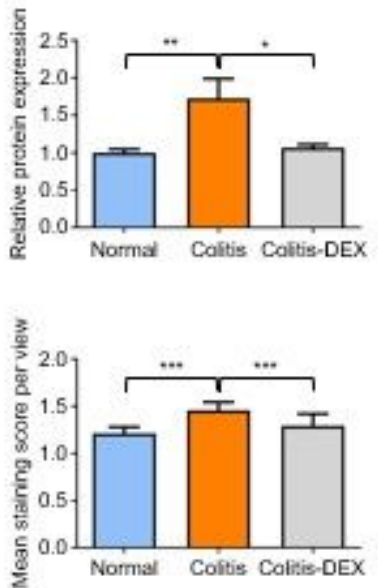

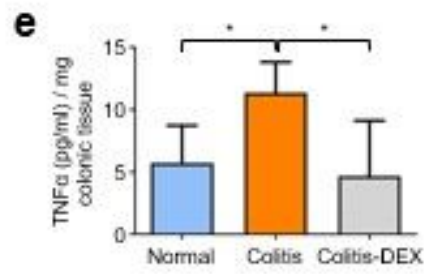

h
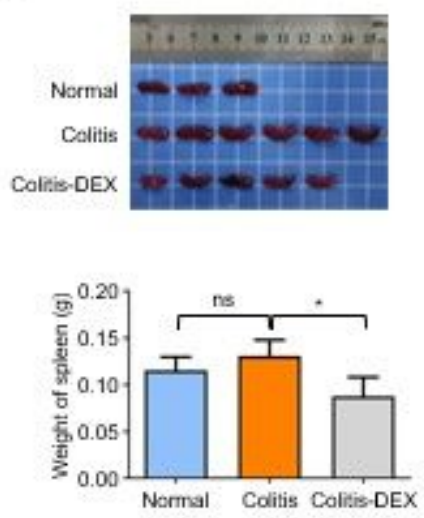

\section{Figure 5}

Inhibitory effect of DEX treatment on DSS-induced colitis in mice. a, The scheme shows the process of DSS-induced colitis $(n=5)$ and DEX intervention $(n=6)$ in mice. b, The representative images of colonic epithelium of normal, colitis and DEX-treated mice (H\&E staining, 200x amplification). c, The local inflammation was histologically scored by lymphocytes infiltration, mucosal injury, crypt damage and lesion extent in Normal ( $n=3)$, Colitis $(n=6)$ and Colitis-DEX $(n=5)$ mice. $d$, The infiltration of macrophages in epithelial tissues was measured by IHC staining with F4/80 antibody (left panels, $n=3$ per group). Statistical results were presented in right panel. e, The TNFa production was measured by ELSA and normalized by weight of tissue specimens in Normal ( $n=3)$, Colitis $(n=4)$ and Colitis-DEX ( $n$ =3) mice. $f$, TNFa expression was evaluated in colonic tissues of the indicated mice by western blot analysis (Left panels, $n=3$ per group) and measured using densitometry. $\beta$-action was used as a loading control. Statistical results were presented in right panel. $\mathrm{g}$, Left panels show the representative IHC images of TNFa staining in colonic epitheliums in the indicated mice ( $n=3$ per group). Statistical results were presented in right panel. $h$, The spleen images (upper panel) and statistical results of spleen weight (lower panel) in Normal $(n=3)$, Colitis $(n=6)$ and Colitis-DEX $(n=5)$ mice. The scale represents $100 \mu \mathrm{m}$. All data were expressed as mean $\pm S D$. * $P<0.05$; **, $P<0.01$; ***, $P<0.001$; ns, not significant. 
a

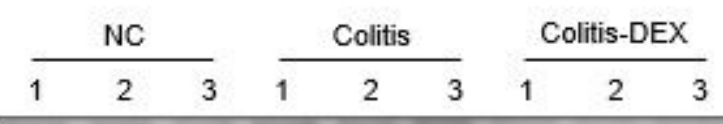

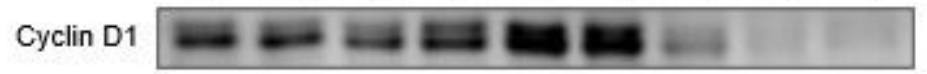

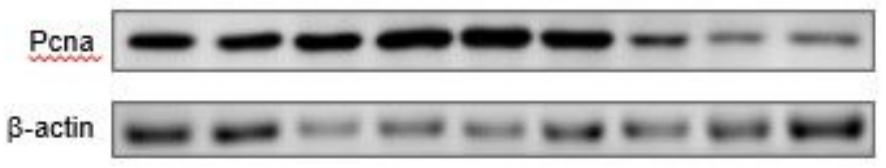

b

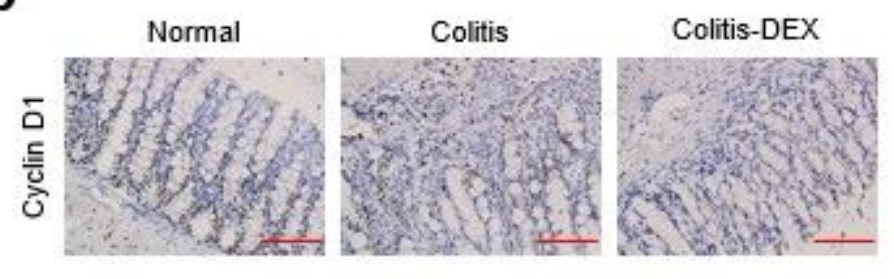

C

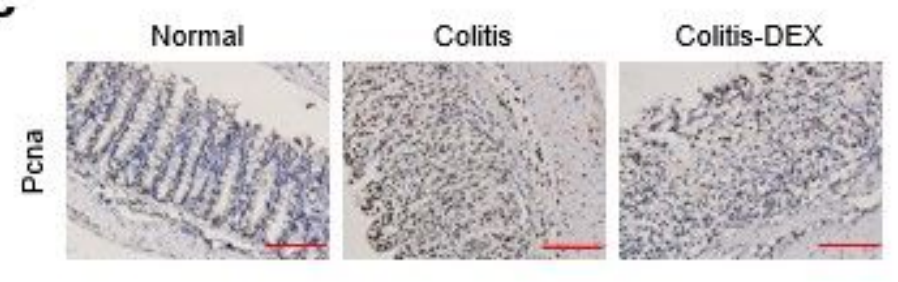

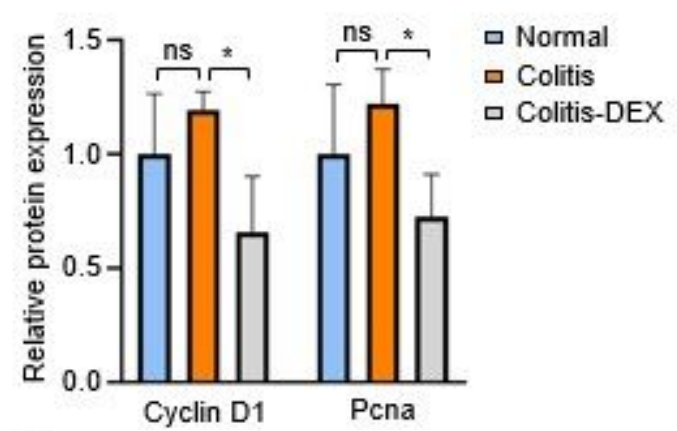
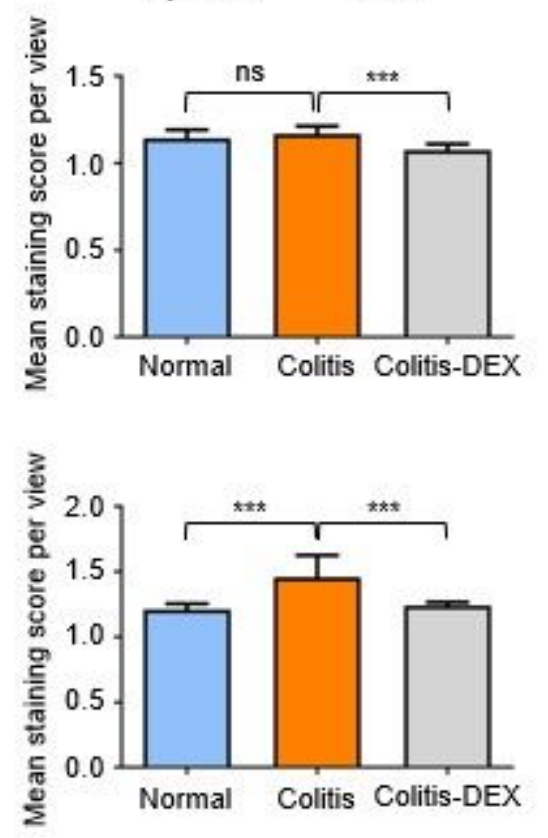

Figure 6

DEX treatment inhibits the expression of cyclinD1 and Pcna in DSS-induced colitis mice. a, The expression of proliferative markers cyclinD1 and Pcna was evaluated in colonic tissues of the indicated mice by western blot analysis and measured using densitometry (left panel, $n=3$ per group). $\beta$-action was used as a loading control. Statistical results were presented in right panel. Left panels show the representative IHC images (400x) of cyclinD1 (b) and Pcna (c) staining in colonic epitheliums in the indicated mice ( $n=3$ per group). Statistical results were presented in right panel. The scale represents 100 $\mu \mathrm{m}$. Data were expressed as mean $\pm \mathrm{SD}$. *, $\mathrm{P}<0.05$; $* \star \star, P<0.001$; ns, not significant. 

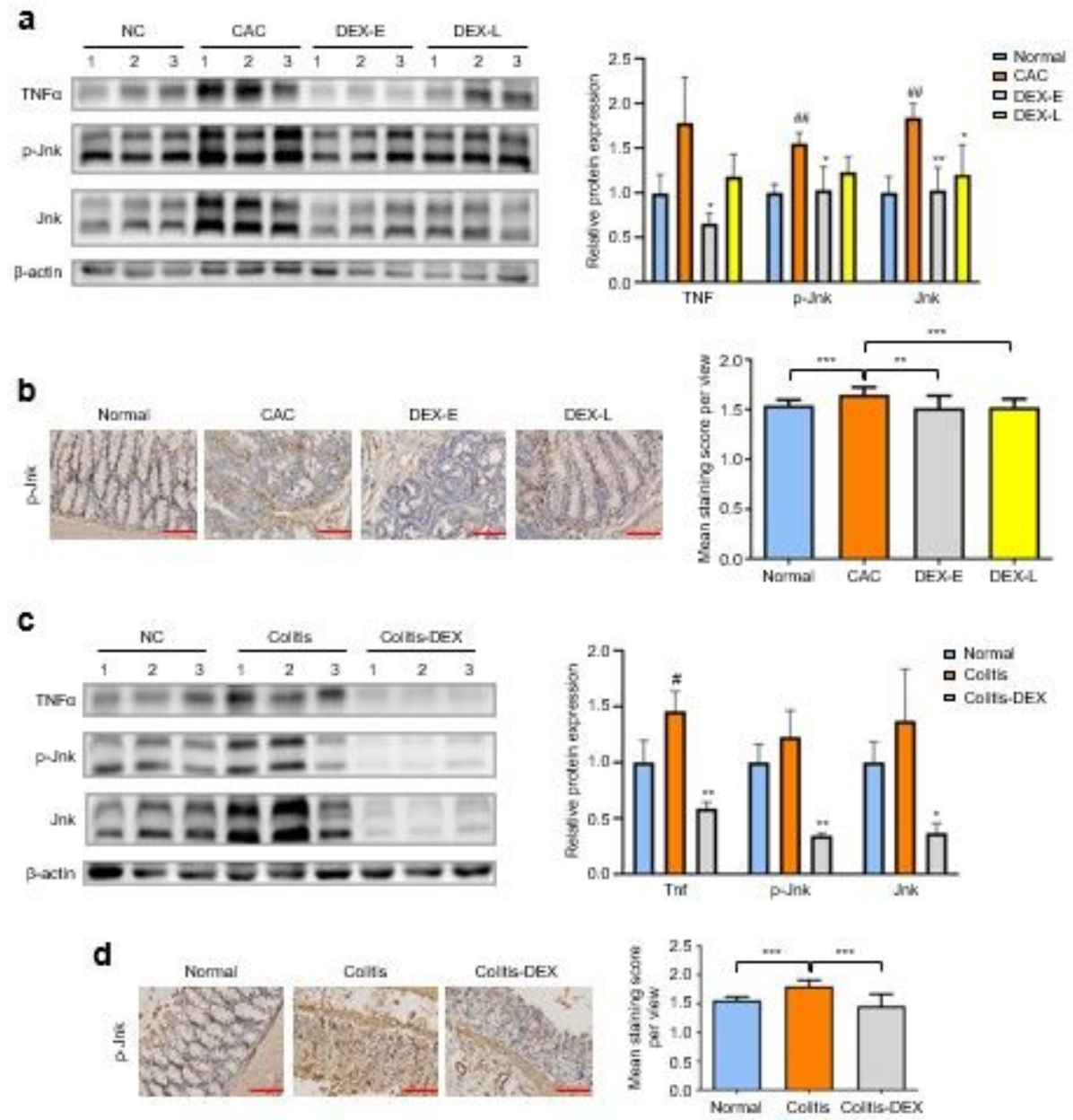

\section{Figure 7}

DEX treatment inhibits the activity of MAPK/JNK pathway. a, The levels of Jnk and p-Jnk in tumor tissues of AOM/DSS-treated mice and colonic tissues of normal mice were measured by western blot analysis and measured using densitometry (left panel, $n=3$ per group). $\beta$-action was used as a loading control. Statistical results were presented in right panel. \#\#, $\mathrm{P}<0.01$ for comparison with Normal; *, $\mathrm{P}<0.05$; **, $\mathrm{P}$ $<0.01$ for comparison with CAC. b, Left panels show the representative IHC images of p-Jnk staining in neoplasms or normal epitheliums of the indicated mice ( $n=3$ per group). Statistical results were presented in right panel. c, The levels of Jnk and p-Jnk were measured in colonic specimens of the indicated mice by western blot analysis and measured using densitometry (left panel, $n=3$ per group). $\beta$ action was used as a loading control. Statistical results were presented in right panel. \#, $P<0.05$ for comparison with Normal; * $\mathrm{P}<0.05$; $* \star, \mathrm{P}<0.01$ for comparison with CAC. $d$, Left panels show the representative IHC images of $p$-Jnk staining in colonic epitheliums of the indicated mice ( $n=3$ per group). Statistical results were presented in right panel. The scale represents $100 \mu \mathrm{m}$. All data were expressed as mean \pm SD. $* \star, ~ P<0.01 ; * \star \star, ~ P<0.001$. 
a

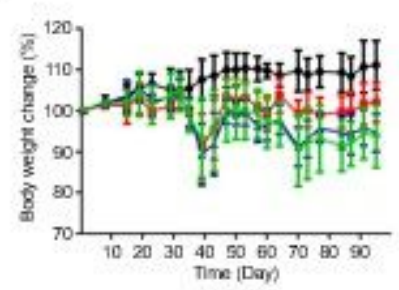

C

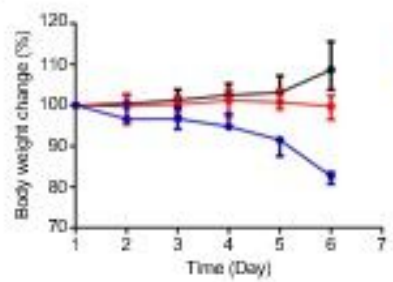

e

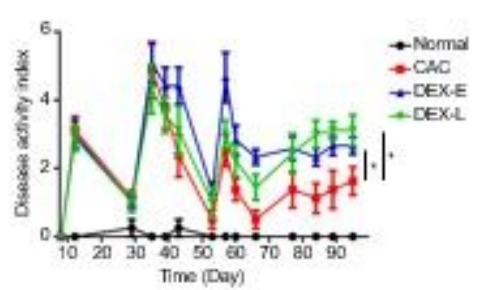

g

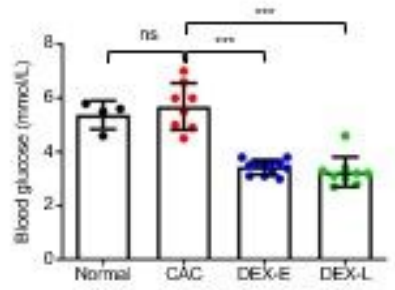

b

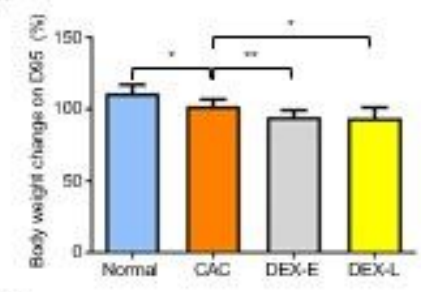

d

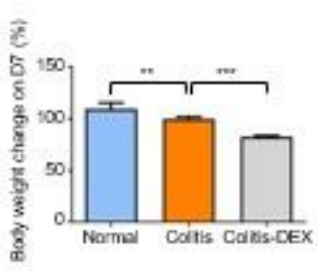

f

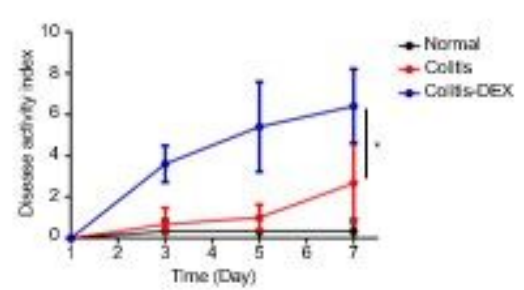

h

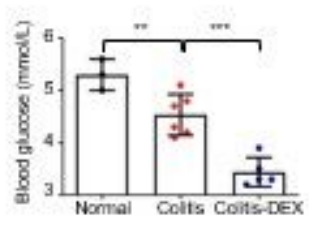

\section{Figure 8}

DEX administration deteriorates the general state of mice. a, The effect of DEX treatment $(5 \mathrm{mg} / \mathrm{kg})$ on body weight of Normal $(n=4), C A C(n=6), D E X-E(n=12)$ and DEX-L $(n=9)$ mice. b, Body weight change of Normal $(n=4), C A C(n=6), D E X-E(n=12)$ and DEX-L $(n=9)$ mice on D95. $c$, The effect of DEX treatment $(5 \mathrm{mg} / \mathrm{kg})$ on body weight of Normal $(n=3)$, Colitis $(n=6)$ and Colitis-DEX $(n=5)$ mice. $d$, Body weight change of Normal $(n=3)$, Colitis $(n=6)$ and Colitis-DEX $(n=5)$ mice on D7. e, Disease activity index (DAl) of Normal $(n=4), C A C(n=6)$, DEX-E $(n=12)$ and DEX-L $(n=9)$ mice, scored by loss of body weight, rectal bleeding and stool consistency. $f$, Disease activity index (DAl) of Normal $(n=3)$, Colitis $(n=6)$ and ColitisDEX $(n=5)$ mice. $g$, The fasting glucose of Normal $(n=4)$, CAC $(n=6)$, DEX-E $(n=12)$ and DEX-L $(n=9)$ mice at the end of the experiment. $h$, The fasting glucose of Normal $(n=3)$, Colitis $(n=6)$ and Colitis-DEX $(n=5)$ mice on D7 at the end of experiment. Data were expressed as mean \pm SD. ${ }^{*}, P<0.05$; $* \star, P<0.01$; $\star \star \star, P<0.001 ; n s$, not significant.

\section{Supplementary Files}

This is a list of supplementary files associated with this preprint. Click to download. 
- Additionalfile1.docx

Page 24/24 